\title{
Dialisi peritoneale e strategie per la diffusione: le strade del Piemonte; analisi critica dei risultati e apertura di nuovi fronti di intervento
}

\author{
Gian Maria Iadarola ${ }^{1}$, Mario Salomone ${ }^{2}$, Stefano Maffei $^{3}$, Loris Neri ${ }^{4}$ \\ ${ }^{1}$ Nefrologia e Dialisi, Ospedale S. Giovanni Bosco, ASL TO2, Torino \\ ${ }^{2}$ Nefrologia e Dialisi, Ospedale Maggiore, ASL TO5, Chieri (TO) \\ ${ }^{3}$ Nefrologia e Dialisi, Azienda Ospedaliera CTO/Maria Adelaide, Torino \\ ${ }^{4}$ Nefrologia e Dialisi e Nutrizione Clinica, Ospedale San Lazzaro, ASL CN2, Alba (CN)
}

\begin{abstract}
Peritoneal dialysis and strategies for its diffusion: the Solutions of Piedmont Region; Critical analysis of the Results AND NEW FRONTIERS

Abstract. The well-known phenomenon of gradual reduction of patients undergoing haemodialysis (HDD) or peritoneal dialysis (PD) treatment at home seems to be caused by aging of incident patients, comorbilities, loneliness, and the presence of cultural and organizational obstacles. A measure approved by the Piedmont region (D.G.R. 8-12316 - October 12, 2009) provides a financial contribution supporting home dialysis (HoD). The amount of the contribution depends on a scoring system (Home Dialysis Assistance Plan - P.A.I.D.D.) assessing each patient for his lack of independence in managing the treatment. The final score identifies the patients' assistance needs and, thus, the amount of financial support, also according to the type of treatment and caregiver. At the end of the first 3-year period of use of the measure, the overall population on dialysis was stable; incidence and prevalence of HoD patients increased in 2010 and remained stable in the period 2011-2012. It is possible that this measure can effectively reverse the incidence and prevalence trend of HoD patients: in fact, it turned out to be a potentially powerful tool, despite not being sufficient by itself. Analysis of further means to integrate this measure are currently undergoing in Piedmont, in order to facilitate the solution of other problems, including the poor motivation of nefrologists, prejudice, and the lack of knowledge and background on the issue of home dialysis.
\end{abstract}

Key words: Home dialysis, Peritoneal dialysis, Home haemodialysis, Assisted dialysis, Lack of indipendence, Home dialysis trend, PAIDD, Piedmont, Financial contribution

Conflict of interest: None.

Financial support: None.

Accettato: 27 Ottobre 2013

In una recente analisi dei dati ricavati dal Censimento SIN (1), Viglino ha evidenziato come il ridotto utilizzo della dialisi peritoneale (DP) in Italia sia riconducibile in ampia parte a fattori organizzativi e a politiche del centro, ovvero: 1) natura pubblica o privata del centro, 2) dimensioni del centro dialisi, 3) disponibilità di posti letto di HD e, ultima cosa, ma non per importanza, 4) fattori culturali.

Non sembra secondario, d'altra parte, l'impatto sulla diffusione della metodica di un altro fattore, comune alla maggior parte dei paesi occidentali, ovvero il progressivo invecchiamento della popolazione incidente in dialisi (2-4).

I dati del Registro Italiano di Dialisi e Trapianto (RIDT) (in modo concorde con quelli dei maggiori registri del mondo oc- cidentale) riportano, infatti, come la probabilità di un soggetto di essere avviato alla dialisi peritoneale sia inversamente proporzionale alla sua età (5), ed è, pertanto, evidente come la diffusione di un trattamento autogestito come la DP risulti influenzato negativamente dal progressivo incremento delle comorbidità e dall'isolamento sociale (e, quindi, da una minore autonomia), associati all'invecchiamento (6). Anche Mehrotra, in un'analisi del 2007 (7), riportava come, negli USA, la minore penetrazione della dialisi peritoneale non risulterebbe conseguente a un'influenza preponderante di possibili controindicazioni cliniche, bensì a un impatto critico di cause economiche, strutturali e culturali.

A questo proposito, Oliver (8), in un ben noto lavoro del 2010, 


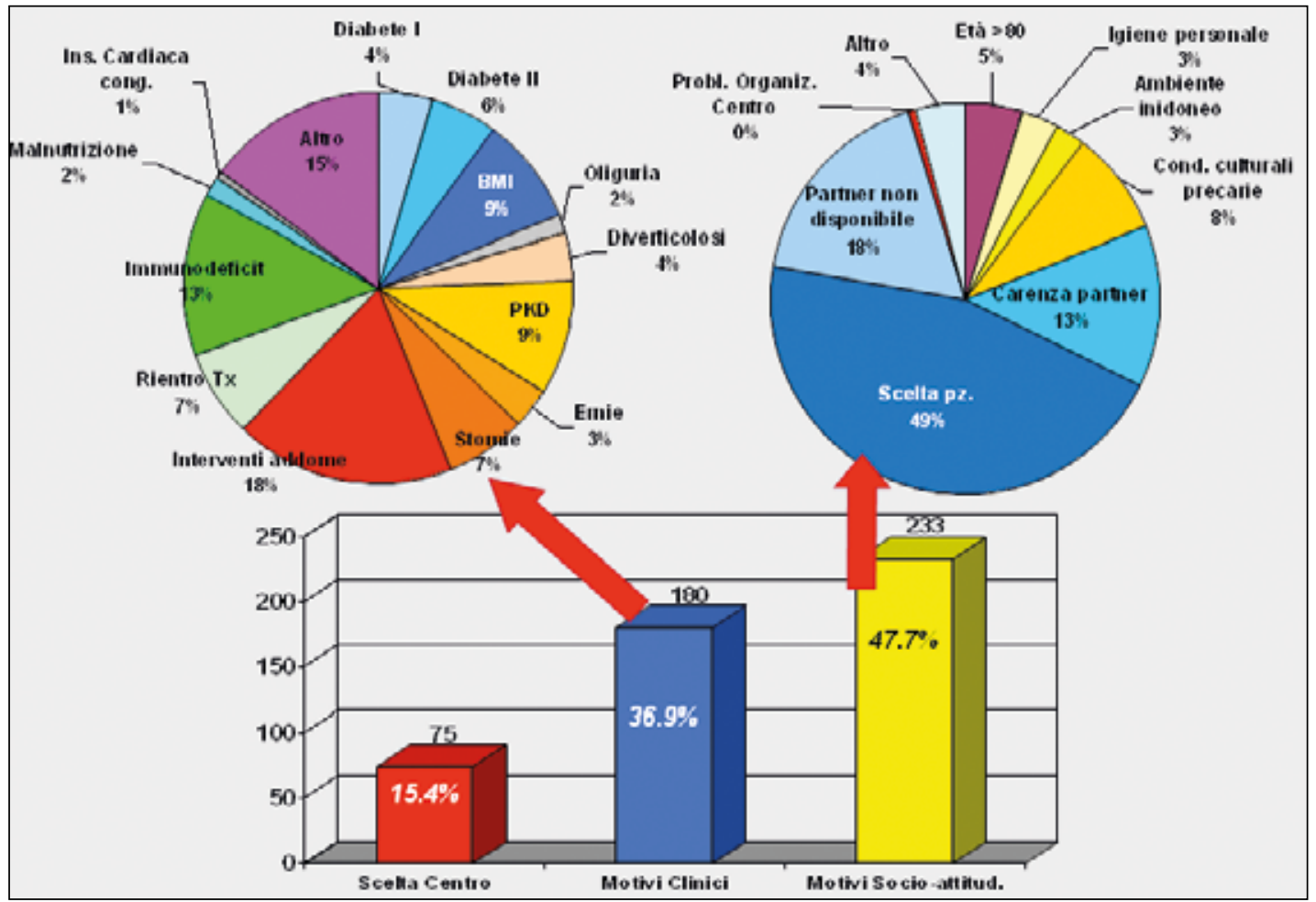

Fig. 1 - Motivi di non ammissione in DP (Primo Audit Piemontese sulla Dialisi Peritoneale, 2009, $465 \mathrm{pz}$ incidenti in DP con età $\geq 18$ anni in trattamento presso 23 centri dialisi della Regione Piemonte).

ha messo in evidenza come, su 497 pz incidenti con insufficienza renale cronica terminale, solo 110 (22.13\%) presentassero controindicazioni cliniche alla DP mentre, dei restanti $387 \mathrm{pz}(77.87 \%)$ potenzialmente eleggibili alla DP, ben 245 (49.29\%) presentassero problematiche, relative all'autonomia, ostacolanti l'autogestione. La crescente solitudine, associata alla carenza di eventuali caregiver o alla non disponibilità degli stessi, si confermerebbe essere un ulteriore elemento limitante l'utilizzo della DP negli anziani: l'estendersi progressivo di aree abitative di tipo metropolitano, accompagnandosi a differenti necessità lavorative dei membri attivi delle famiglie, ha parcellizzato la presenza familiare sul territorio, riducendo ulteriormente le possibilità di assistenza nei confronti dei familiari anziani.

Limitare, però, le cause della ridotta diffusione della dialisi peritoneale in Italia al solo impatto delle cause sociali e della natura e della politica organizzativa del centro dialisi, oltre a fattori culturali da parte dei pz, è probabilmente insufficiente per descrivere completamente il problema. Un elemento di estrema importanza sembra essere, infatti, rappresentato dalla modesta motivazione nei confronti della dialisi peritoneale da parte dei nefrologi, in parte condizionata, spesso e volentieri, da stanchezza professionale, pregiudizi e insufficienti conoscenza e preparazione culturale rispetto alla metodica, tutte problematiche, peraltro, già analizzate in letteratura (9), senza che, nel tempo, si siano trovati correttivi efficaci.

La realtà piemontese si adatta, per le sue caratteristiche, a questo scenario: i dati del Registro Piemontese di Dialisi e Trapianto (RPDT), evidenziano, infatti, come vi sia stato un progressivo declino della DP, la cui prevalenza è scesa dal $18.8 \%$ del 1997 all'11.5\% del 2009 (10), nonostante i dati di sopravvivenza in DP fossero sovrapponibili a quelli in emodialisi ospedaliera, con un andamento in linea con quanto riportato nella letteratura internazionale dai maggiori Autori, in termini di sopravvivenza e qualità della vita di pz $(11,12)$, che riferiscono, peraltro, anche dati positivi relativi alla qualità di vita dei pz e dei loro caregiver (13).

Anche l'Audit Piemontese sulla Dialisi Peritoneale del 2009 (10) ha messo in luce l'importanza delle problematiche di tipo non clinico come barriere a un utilizzo più aperto della DP (Fig. 1), con dati assai simili a quelli riportato in letteratura per realtà analoghe, permettendo di individuare la situazione di questa Regione del nord Italia come potenzialmente paradigmatica di quella di buona parte dei paesi occidentali e come realistico laboratorio per la ricerca di correttivi a sostegno delle metodiche dialitiche domiciliari. I dati messi a disposizione dall' Audit mostrano, infatti, un forte impatto, oltre che delle ben note problematiche sociali e culturali, anche delle politiche organizzative e delle dimensioni del centro, a conferma di quanto riportato da Viglino: in oltre il $60 \%$ dei casi, la scelta di non immissione del pz in un programma di dialisi domiciliare non era dovuta, in Piemonte, a motivi di tipo clinico.

Sulla base di questi dati, i nefrologi piemontesi, tenuto conto anche di altre esperienze europee sul tema, delle loro criticità e delle proposte critiche della letteratura internazionale, hanno sviluppato un modello di "dialisi domiciliare assistita", basato sul riconoscimento del caregiver, visto come figura cardine del trattamento domiciliare nei casi di ridotta autonomia gestionale del pz.

Essendo il Piemonte una "Regione in piano di rientro dal debito sanitario", nell'elaborazione del modello sono stati tenuti in conto anche l'aspetto economico dei costi dialitici (Censis 
2009) (14) e un'analisi dei costi nei pz in trattamento dialitico. Con la DGR 8-12316 del 12 Ottobre del 2009 "Potenziamento delle cure domiciliari nei pz affetti da insufficienza renale terminale con necessità di trattamento dialitico tramite un contributo economico di sostegno alla Dialisi Domiciliare" (15), frutto di un lavoro congiunto tra le istituzioni amministrative sanitarie e un tavolo di lavoro multidisciplinare a forte componente nefrologica, la Regione Piemonte ha istituito un contributo economico sperimentale a sostegno della de-ospedalizzazione della dialisi. Al centro del provvedimento vi è la Commissione Nefrologica Aziendale, composta dal Direttore del centro, dal Medico e dall'Infermiera di DP (e HDD) e dall'Assistente Sociale. Compito della Commissione è la stesura del Piano Assistenziale per la Dialisi Domiciliare (P.A.I.D.D.), con il quale viene valutata, mediante un sistema di punteggi (codificati da tabelle di riferimento), la disautonomia del pz per quanto riguarda le necessità gestionali di base del trattamento (medicazione exit site, rilevazione peso, pressione arteriosa e igiene personale e ambientale) e l'esecuzione del trattamento stesso (cambi DP manuali, gestione DP automatizzata). Il punteggio totale definisce l'intensità assistenziale del pz (bassa, media, alta) e, quindi, il sostegno economico, differenziato ulteriormente in base alle caratteristiche del caregiver (Assistente Familiare, Familiare, Affidatario). All'esordio, l'impianto della DGR era affiancato da obiettivi rivolti ai Direttori Generali delle Aziende Sanitarie piemontesi, con l'indicazione di un tasso di incidenza minima annuale, auspicato per le metodiche dialitiche domiciliari.

L'analisi dei dati di incidenza e prevalenza (Tabb. I, II) a tre anni dall'avvio dell'applicazione della DGR mostra un andamento caratterizzato da un risultato positivo nella prima fase (soprattutto nel 2010) e seguito da una successivo appiattimento della curva di crescita, rispetto al quale incide almeno in parte una sostanziale stabilizzazione/flessione del numero totale di pz in dialisi nella Regione.

L'incremento maggiore (circa $+1.6 \%$ di pz prevalenti in più in dialisi domiciliare) è stato sicuramente legato alla presenza di un obiettivo per i Direttori Generali della Regione, teso a portare fino al $20 \%$ il numero di nuovi ingressi in dialisi domiciliare sul totale degli ingressi nell'anno

L'obiettivo che ci si era inizialmente posti $(+5 \%$ di prevalenza in 3-4 anni) non è si è dimostrato raggiungibile.

$\mathrm{Se}$, da una parte, l'esperienza acquisita in questi tre anni ci mostra come, con l'avvio del sostegno economico previsto dalla DGR, si sia fornito ai nefrologi uno strumento (riteniamo importante) per ovviare a buona parte delle problematiche sociali legate alla scelta del trattamento dialitico domiciliare, dall'altra, dimostra come lo strumento da solo non sia sufficiente quando vengono a mancare la motivazione individuale del nefrologo e il cambiamento delle politiche organizzative del centro anche come necessità di raggiungere obiettivi regionali (questi ultimi mantenuti in vigore solo nel primo anno). $\mathrm{Ci}$ sono, poi, da considerare le problematiche costituite da carenza di pratica clinica e di risorse generate dalle dimensioni del centro.

L'estrema variabilità dell'applicazione della DGR e la forte variabilità dei risultati in termini di incidenza e prevalenza dei pz in dialisi peritoneale presso i vari centri della Regione sono paradigmatiche della situazione. L'avvento della DGR ha permesso di arrestare il declino della dialisi domiciliare nella Regione, invertendo una tendenza ormai inveterata negli ultimi anni e ponendo un freno alla necessità di nuovi posti

TABELLA I - PREVALENZA DEI PZ IN TRATTAMENTO DIALITICO IN REGIME DOMICILIARE (DP E HDD) E DEI TRATTAMENTI DIALITICI IN REGIME OSPEDALIERO (HD) AL 31-12-2009, 2010, 2011 E 2012 NEI CENTRI DIALISI DELLA REGIONE PIEMONTE

\begin{tabular}{|c|c|c|c|c|c|}
\hline & & 2009 & 2010 & 2011 & 2012 \\
\hline \multirow[t]{3}{*}{$\mathrm{Pz}$ in trattamento dialitico domiciliare } & Totale & 359 & 394 & 412 & 385 \\
\hline & Dialisi Peritoneale & 347 & 382 & 401 & 374 \\
\hline & Emodialisi domic. & 12 & 12 & 11 & 11 \\
\hline $\mathrm{Pz}$ in trattamento dialitico ospedaliero & Totale & 2790 & 2766 & 2745 & 2737 \\
\hline Pz in trattamento dialitico in Reg. Piemonte & Totale & 3149 & 3160 & 3157 & 3122 \\
\hline
\end{tabular}

TABELLA II - INCIDENZA DEI PZ IN TRATTAMENTO DIALITICO IN REGIME DOMICILIARE (DP E HDD) E DEI TRATTAMENTI DIALITICI IN REGIME OSPEDALIERO (HD) AL 31-12-2009, 2010, 2011 E 2012 NEI CENTRI DIALISI DELLA REGIONE PIEMONTE

\begin{tabular}{|c|c|c|c|c|c|}
\hline & & 2009 & 2010 & 2011 & 2012 \\
\hline \multirow[t]{3}{*}{$\mathrm{Pz}$ in trattamento dialitico domiciliare } & Totale & 135 & 181 & 179 & 154 \\
\hline & Dialisi Peritoneale & 134 & 179 & 178 & 151 \\
\hline & Emodialisi domic. & 1 & 2 & 1 & 3 \\
\hline $\mathrm{Pz}$ in trattamento dialitico ospedaliero & Totale & 660 & 635 & 664 & 637 \\
\hline $\mathrm{Pz}$ in trattamento dialitico in Reg. Piemonte & Totale & 795 & 816 & 843 & 791 \\
\hline
\end{tabular}


tecnici per l'emodialisi ospedaliera, con i relativi costi legati alle strutture e al personale.

Sono attualmente all'analisi provvedimenti di "tuning" del dispositivo, compresa l'evidente necessità di porre nuovamente obiettivi di incidenza e prevalenza, oltre che di avviare la costituzione di una rete ad hoc per la diffusione di conoscenze e risorse tecniche per la dialisi domiciliare tra i vari centri nefrologici della Regione.

Fin da quando abbiamo avviato il tavolo di lavoro per la stesura della DGR per il sostegno alla domiciliarità, abbiamo ritenuto importante associare a questa azione altri interventi tesi a stimolare la Nefrologia della Regione a considerare il trattamento sostitutivo domiciliare. Al di là dell'obiettivo istituzionale, abbiamo riunito, sotto l'egida dell'ARESS (Agenzia Regionale Servizi Sanitari) e su mandato dell'assessorato alla salute, un gruppo di esperti in dialisi peritoneale e in dialisi domiciliare, che ha portato alla preparazione di un percorso diagnostico terapeutico assistenziale (PDTA) sull'avvio del trattamento sostitutivo. In quel PDTA, si individuava, nella strutturazione di un ambulatorio per la malattia renale avanzata (ambulatorio Ma.Re.A), il momento essenziale per dare una corretta informazione al pz sul trattamento sostitutivo. L'ambulatorio Ma.Re.A segue un percorso di diagnosi e terapia per pz con stadio 4-5 di CKD, indirizzando il pz a un'iscrizione "precoce" in lista di attesa di trapianto renale, e, nel contempo, permette di dare una corretta e ampia informazione al pz riguardo alla dialisi domiciliare, anche in relazione al "timing" della progressione dell'insufficienza renale.

Il PDTA è stato deliberato lo scorso anno (DGR n. 59-2569 del 12 Marzo del 2013) (16) dalla giunta regionale.

La deliberazione del PDTA sull'avvio del trattamento sostitutivo ha permesso di avviare un'azione di sensibilizzazione dell'assessorato regionale a questa problematica, con il risultato di giungere in tempi recentissimi all'approvazione della DGR 88-6290 del 2 Agosto del 2013 (17), in cui viene normata l'attività degli ambulatori Ma.Re.A.

Riteniamo che questo tipo di ambulatorio, se ben organizzato, possa rappresentare il momento fondamentale per ottimizzare il processo di avvio al trattamento dialitico, con la possibilità, da una parte, di incrementare il processo di avvio al trapianto renale anche preventivo per i pz eleggibili al trapianto renale e, dall'altra, di incentivare il processo di avvio alle diverse metodiche di dialisi domiciliare.

Il sistema è stato testato dai nefrologi piemontesi nei due anni passati (13 centri/24 coinvolti nella sperimentazione) e, a breve, potremo ricavare i primi dati su questa esperienza.

Al momento, i dati preliminari sembrano dimostrare che l'attivazione degli ambulatori ha permesso di ridurre il tempo necessario per l'iscrizione in lista di attesa trapianto. Altro dato interessante è quello della riduzione del numero di ricoveri annuali e della relativa spesa.

Riteniamo che un lavoro di "training" dei medici attivi presso i diversi ambulatori Ma.Re.A. della Regione possa portare a un incremento del numero di pz avviati alla dialisi domiciliare e peritoneale, conducendo anche a una maggiore consapevo- lezza riguardo alle varie possibilità di sostegno al pz offerte dal supporto economico per la dialisi domiciliare previsto dalla DGR 8-12316 del 12 Ottobre del 2009.

\section{Riassunto}

Si ritiene che il ben noto fenomeno della progressiva diminuzione dei pazienti (pz) sottoposti a emodialisi (HDD) o a dialisi peritoneale (DP) domiciliare sia determinato da età avanzata dei pz incidenti, comorbidità, solitudine e presenza di ostacoli culturali e organizzativi.

Un provvedimento approvato dalla Regione Piemonte (DGR 8-12316, del 12 Ottobre del 2009) stabilisce un contributo finanziario a sostegno della dialisi domiciliare (HDDom), la cui entità deriva da un sistema di valutazione a punti (Piano Assistenziale Individuale Dialisi Domiciliare, P.A.I.D.D.), che valuta la ridotta autonomia del pz nel gestire il trattamento. Il punteggio finale definisce l'intensità della necessità assistenziale e, quindi, il sostegno finanziario calcolato anche in base al tipo di trattamento e alla tipologia del caregiver. Al termine del primo triennio, il numero dei pz della popolazione totale in trattamento dialitico in Regione era stabile; incidenza e prevalenza dei pz HDDom sono aumentate nel 2010 e risultano stabili nel 2011-2012. È verosimile che il provvedimento si sia dimostrato efficace nell'invertire la tendenza di incidenza e prevalenza dei pz HDDom: lo stesso si rivela essere uno strumento potente, ma non sufficiente di per sé. In Piemonte, attualmente, sono allo studio altre soluzioni per integrare il provvedimento, al fine di agevolare la soluzione di altri problemi, tra cui la scarsa motivazione dei nefrologi, i pregiudizi e la mancanza di conoscenza e di preparazione culturale in merito alla dialisi domiciliare.

Parole chiave: Dialisi domiciliare, Dialisi peritoneale, Emodialisi domiciliare, Dialisi assistita, Perdita di autonomia, Andamento dialisi domiciliare, P.A.I.D.D., Piemonte, Sostegno economico

Dichiarazione di conflitto di interessi: Gli Autori dichiarano di non avere conflitto di interessi.

Contributi economici degli autori: Gli Autori dichiarano di non aver ricevuto sponsorizzazioni economiche per la preparazione dell'articolo.

Indirizzo degli Autori:

Dr. Gian Maria Iadarola

S.C. Nefrologia e Dialisi

Ospedale San G. Bosco - ASL TO2

P.zza Donatore di Sangue, 3

10154 Torino

gianmaria.iadarola@aslto2.it 


\section{Bibliografia}

1. Viglino G, Neri L, Alloatti S, et al. Analysis of the factors conditioning the diffusion of peritoneal dialysis in Italy. Nephrol Dial Transplant 2007; 22 (12): 3601-5.

2. Limido A, Nichelatti M, Postorino M, et al. Come cambia la popolazione uremica nel Registro Nazionale di Dialisi e Trapianto. G Ital Nefrol 2012; 29 (Suppl. 58): S21-6.

3. U.S. Renal Data System, USRDS 2012 Annual Data Report: Atlas of Chronic Kidney Disease and End-Stage Renal Disease in the United States, National Institutes of Health, National Institute of Diabetes and Digestive and Kidney Diseases, Bethesda, MD 2012.

4. Canadian Institute for Health Information, Canadian Organ Replacement Register Annual Report: Treatment of End-Stage Organ Failure in Canada 2001 to 2010 (Ottawa, Ont.: CIHI, 2011).

5. Società Italiana di Nefrologia, Report 2010 del Registro Italiano di Dialisi e Trapianto. http://www.sin-ridt.org/web/eventi/ RIDT/registro_italiano.cfm

6. van Biesen W, Veys N, Lameire N, Vanholder R. Why less success of the peritoneal dialysis programmes in Europe? Nephrol Dial Transplant 2008; 23: 1478-81.

7. Mehrotra R, Kermah D, Fried L, et al. Chronic peritoneal dialysis in the United States: declining utilization despite improving outcomes. J Am Soc Nephrol 2007; 18 (10): 2781-8.

8. Oliver MJ, Garg AX, Blake PG, et al. Impact of contraindications, barriers to self-care and support on incident peritoneal dialysis utilization. Nephrol Dial Transplant 2010; 25 (8): 2737-44.
9. Gabella P. Pregiudizi alla diffusione della dialisi peritoneale in Italia. GTN\&D 2001; 13 (Suppl. 1): 35-6.

10. Maffei S, Iadarola GM, Neri L, et al. Indicazioni dal primo Audit sulla Dialisi Peritoneale in Piemonte e Valle d'Aosta. [Indications from the first audit on peritoneal dialysis in Piedmont and Aosta valley]. G Ital Nefrol 2011; 28 (2): 188-94.

11. Harris SA, Lamping DL, Brown EA, Constantinovici N. Clinical outcomes and quality of life in elderly patients on peritoneal dialysis versus hemodialysis. Perit Dial Int 2002; 22 (4): 463-70.

12. Brown EA, Johansson L, Farrington K, et al. Broadening Options for Long-term Dialysis in the Elderly (BOLDE): differences in quality of life on peritoneal dialysis compared to haemodialysis for older patients. Nephrol Dial Transplant 2010; 25 (11): 3755-63.

13. Belasco A, Barbosa D, Bettencourt AR, et al. Quality of life of family caregivers of elderly patients on hemodialysis and peritoneal dialysis. Am J Kidney Dis 2006; 48: 955-63.

14. Le ricerche della Fondazione CENSIS. I trattamenti sostitutivi della funzione renale in Italia. Aspetti clinici, economici e sociali. 3 Marzo 2009.

15. Regione Piemonte, D.G.R. n. 8-12316 del 12/10/09 - Bollettino Ufficiale Regione Piemonte n. 43 del 29/10/09.

16. Regione Piemonte, D.G.R. n. 59-3569 del 12/03/12 - Bollettino Ufficiale Regione Piemonte n. 16 del 19/04/12.

17. Regione Piemonte D.G.R. n. 88-2690 del 2/08/13 - Bollettino Ufficiale Regione Piemonte n. 36 del 5/09/13. 